\title{
Société Française de Physique, Société Française d’Optique, un rapprochement nécessaire
}

L'année 2015 a vu la signature par les présidents de la SFP et de la SFO d'un accord-cadre dans le but de coordonner les activités des deux sociétés savantes (voir Reflets de la physique $n^{\circ} 43$, p. 54, et n46, p. 37). Il s'agissait d'amplifier la visibilité de nos deux sociétés, d'augmenter l'impact des actions concertées auprès de nos interlocuteurs, pour la plupart communs, organismes de recherche, universités et partenaires industriels. Une cotisation globale réduite pour l'adhésion groupée à la SFP et à la SFO a été mise en place dès 2016, afin de donner aux membres des deux sociétés savantes le sentiment d'appartenir à une communauté élargie, capable de produire des actions communes. Au niveau de la gouvernance, chaque conseil d'administration accueille en son sein un membre du conseil d'administration de la société savante sœur.

Plusieurs actions concrètes ont été mises en place depuis lors.

La première a porté sur la coordination des calendriers des deux congrès nationaux, afin d'éviter des concurrences passives qui desservent les communautés scientifiques concernées et les entreprises adhérentes soutenant leur dynamique. Ces congrès sont maintenant organisés en alternance, les années impaires pour la SFP et les années paires pour la SFO. Chaque société savante prend alors soin d'aménager un espace d'accueil visible, ouvert à la société sœur. Ce déphasage a été mis en place dès 2016 avec la tenue du congrès OPTIQUE Bordeaux 2016, qui a accueilli les sessions scientifiques de la division de Physique Atomique, Moléculaire et Optique (PAMO) de la SFP. Il en a été de même cette année, où les clubs Nanophotonique et Biophotonique de la SFO ont participé au congrès général de la SFP qui s'est tenu à Orsay du 3 au 7 juillet. À cette occasion, la SFO a également partagé son carnet d'adresses industrielles pour aider à l'organisation d'un salon d'exposants.

Une deuxième action a conduit à la création en 2017 d'une commission « Femmes et Physique » au sein de la SFO, analogue à celle de la SFP. L'idée est de travailler ensemble sur cette problématique des inégalités professionnelles hommes/femmes, mais aussi sur d'autres problématiques importantes en sciences dures, en élargissant les groupes de travail sur les deux sociétés. Dans cet esprit, les commissions de la SFP et les Clubs de la SFO travaillant sur des sujets voisins (jeunes chercheurs et chercheuses, enseignement, culture scientifique, entreprises, pays en voie de développement...) sont invités à se rapprocher. Des actions locales communes pourront aussi être envisagées.

Enfin, l'ouvrage La lumière en lumière : du photon à l'Internet, publié en 2016 par EDP Sciences et lauréat du Prix Roberval 2016, a été coordonné et coécrit par des membres de la SFP et de la SFO. II en est de même des différentes brochures qui ont été réalisées dans le cadre de l'Année de la Lumière 2015.

À l'image de toutes ces actions communes, il serait souhaitable que la lumière continuât à rayonner sur ce partenariat SFP-SFO.

Benoît Boulanger, président de la Société Française d’0ptique Olivier Dulieu, responsable de la division Physique Atomique, Moléculaire et Optique de la SFP

Michel Spiro, président de la Société Française de Physique 